Cahiers de philosophie de l'université de

\title{
La question de l'art à la lumière des « Cahiers de travail » de Martin Heidegger ou l'art sur la sellette à l'âge d'une urgente transition
}

Pascal David

\section{(2) OpenEdition}

Journals

Édition électronique

URL : https://journals.openedition.org/cpuc/293

DOI : $10.4000 /$ cpuc. 293

ISSN : 2677-6529

Éditeur

Presses universitaires de Caen

Édition imprimée

Date de publication : 1 novembre 2018

Pagination : 49-74

ISBN : 978-2-84133-904-4

ISSN : $1282-6545$

Référence électronique

Pascal David, « La question de l'art à la lumière des « Cahiers de travail » de Martin Heidegger ou l'art sur la sellette à l'âge d'une urgente transition », Cahiers de philosophie de l'université de Caen [En ligne], 55 | 2018, mis en ligne le 01 novembre 2019, consulté le 02 février 2023. URL : http:// journals.openedition.org/cpuc/293; DOI : https://doi.org/10.4000/cpuc.293

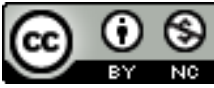

Creative Commons - Attribution - Pas d'Utilisation Commerciale 4.0 International - CC BY-NC 4.0 https://creativecommons.org/licenses/by-nc/4.0/ 


\section{La question de l'art à la lumière des «Cahiers de travail» de Martin Heidegger ou l'art sur la sellette à l'âge d'une urgente transition}

\footnotetext{
J ART SERAIT-IL Ce QU'IL N'y A PLUS ? Mais l'art - qu'est-ce à dire au 1 juste? Telle est la double question posée ici, et que nous allons nous efforcer d'instruire autant que faire se peut à la lumière des «Cahiers de travail» de Heidegger.

Martin Heidegger a soigneusement rédigé entre 1931 et le début des années 1970 des Notizbücher, ou "cahiers de notes", dont une partie est devenue accessible au public en 2014 et 2015 par la publication des tomes 94, 95, 96 puis 97 de la Gesamtausgabe ou édition intégrale de ses écrits chez l'éditeur francfortois Klostermann. Ces quatre cahiers de notes ont tous été édités par Peter Trawny sous le titre contestable et en tout cas controversé de Schwarze Hefte [Cahiers noirs]. Il s'agit d'une publication déjà assez considérable mais encore partielle, qui porte à la connaissance du public 19 des 34 cahiers recensés ou retrouvés, le premier d'entre eux manquant à l'appel. Les cahiers des trois premiers tomes que nous venons de mentionner sont intitulés Überlegungen [Réflexions], ceux du quatrième Anmerkungen [Remarques]. Ces écrits que Heidegger ne voulait voir paraitre, d'après ses propres dispositions testamentaires, qu'une fois publiés tous ses autres écrits dans le cadre de l'édition intégrale, ne viennent pas pour autant «couronner le tout». Ils ne sont ni le "couronnement» de son œuvre ni son «testament philosophique» mais ne sont intelligibles que sur la base d'une connaissance approfondie de tous ses autres écrits, notamment des sept grands traités historiaux rédigés entre 1936 et 1944, à commencer par les Apports à la philosophie. De l'avenance.
} 
Dans l'économie du propos de Heidegger, les «Cahiers de travail» - ainsi les appellerons-nous plus sobrement - accompagnent ces grands traités à titre de compléments et leur sont subordonnés ${ }^{1}$. Ils s'inscrivent dans la perspective de l'histoire de l'être mais ne contribuent pas à élaborer ce qu'il y a de systématique dans l'ajointement interne de la pensée historiale en ses fugues. Les «Cahiers de travail» réaccomplissent le geste de la pensée historiale telle qu'elle s'articule dans les grands traités susdits, à savoir : préparer le passage ou la transition [Übergang] de la pensée métaphysique, ou "premier commencement", parvenu à son terme avec la métaphysique de la subjectivité, à cet «autre commencement » à la faveur duquel est susceptible de s'opérer la métamorphose du sujet en Dasein. Ce qui requiert du sujet qu'il consente à briser la cage dorée de sa subjectivité et de la réduction de l'homme à lui-même. C'est dans l'attente de cet "autre commencement» que l'art se trouve convoqué et interrogé par les «Cahiers de travail». Tout comme c'est dans la perspective de cet «autre commencement» qu'il lui appartient d'être, ou non, inaugural, de trancher ou non, motu proprio, en faveur de cet "autre commencement». La question de l'art à la lumière des «Cahiers de travail», d'une richesse encore largement insoupçonnée, ouvre ainsi un champ considérable, dont notre propos vise simplement à retracer quelques contours et signaler l'ampleur.

C'est surtout à vrai dire à partir du cinquième des «Cahiers de travail» que la question de l'art commence à affleurer, à être abordée et thématisée, c'est-à-dire selon toute vraisemblance à partir du milieu des années 1930 du siècle dernier. C'est-à-dire encore au moment - en 1935 - de la conférence ou plutôt des conférences sur L'origine de l'œuvre d'art. Sous cet intitulé s'annonce la première grande tentative faite par Heidegger pour surmonter l'esthétique. L'art y est envisagé comme mise en œuvre de la vérité dans le jeu contrasté du monde et de la terre dans l'adversité de leur coappartenance. Mais c'est aussi à partir de cette époque que Heidegger prend la mesure de tout ce par quoi l'art de son temps s'enferre toujours davantage dans une métaphysique de la subjectivité qui peu à peu l'asphyxie. D'autant plus pressante se fait dès lors la question de la possibilité d'un «autre commencement» dont l'art pourrait être précurseur s'il parvient à réchapper du régime métaphysique qui a conduit à son épuisement.

Telles seront donc les principales étapes du parcours que nous proposons de suivre ici.

1. Voir à ce sujet l'importante mise au point de F.-W. von Herrmann dans: F.-W. von Herrmann, F. Alfieri, Martin Heidegger. La verità sui "Quaderni neri", Brescia, Morcelliana, 2016, p. 23, dont le lecteur trouvera un résumé dans le volume 33 de la revue Études heideggeriennes, Berlin, Duncker \& Humblot, 2017, p. 303-309. 


\section{Surmonter l'esthétique}

Ces quelques considérations préliminaires étaient nécessaires afin de situer et de mieux circonscrire les propos sur l'art dans les «Cahiers», lorsqu'il arrive à ceux-ci de lui faire un sort. Si l'on se fie aux Indices rerum (et nominum), ou «Index des mots clés» [Stichwortverzeichnisse], que Heidegger a recensés dans les cahiers publiés, le mot Kunst [art] figure dans ceux des Überlegungen V, VI, VII, VIII, IX et des Anmerkungen III et $V$, et fournit leur intitulé à quelques paragraphes. Ces index ne prétendent toutefois à aucune exhaustivité et il convient d'en user avec modération. Heidegger semble en effet les avoir établis afin de retrouver plus facilement tel ou tel passage, mais à son propre usage et pour sa propre gouverne plutôt qu'à l'attention de futurs lecteurs et selon les critères d'une édition «scientifique» en bonne et due forme. Quelque chose d'essentiel peut être dit ou suggéré sur l'art sans que le terme apparaisse expressément, ne serait-ce qu'au détour de passages relatifs à Hölderlin, Goethe, Wagner ou Nietzsche, Rilke, Stefan George, mais aussi Caspar David Friedrich, Thomas Edward Lawrence, Picasso, Braque ou Juan Gris. Allons même jusqu'à risquer cette hypothèse : l'art est omniprésent dans les «Cahiers", il y est présent là même où il n'en est pas expressément question, à la manière de cette note dont Schumann disait qu'elle ne devait pas être jouée mais que l'instrumentiste devait y songer fortement dans l'exécution du morceau. Bref, il y est présent sigétiquement, si inaccessible demeurât-il à toute acoustique comme à toute lexicologie. L'art ne cesse d'être ressource et promesse, là même où il n'est pas thématisé. Nous aimerions montrer ici à quel point ces propos épars et apparemment décousus sur l'art dans les "Cahiers", dont l'aspect rhapsodique tranche avec le caractère plus «systématique» des traités historiaux, sont à la fois limités et fulgurants.

La pensée historiale de l'être que Heidegger appelle «das seinsgeschichtliche Denken» tente de dire, ou plutôt de laisser se dire, la «vérité de l'être» - ou de l'estre [Seyn]. Penser l'estre, c'est "arriver à ce que l'être vienne à la pensée», selon la traduction proposée par François Fédier du verbe erdenken². Penser l'art, ce n'est pas lui extorquer son secret mais le penser hors métaphysique, hors des eaux territoriales de celle-ci, c'est-à-dire en se délivrant des œillères de l'esthétique, qui n'est jamais que «le regard que porte la métaphysique sur le beau et sur l'œuvre d'art» ${ }^{3}$.

2. Voir M. Heidegger, Apports à la philosophie. De l'avenance, F. Fédier (trad.), Paris, Gallimard, 2013, p. 525 et N.D.T., et les remarques de Heidegger lui-même sur l'emploi qu' il fait de ce terme et comment il demande à être entendu dans Besinnung, GA 66, 357.

3. Remarque de J. Beaufret dans: E. de Rubercy, D. Le Buhan, Douze questions posées à Jean Beaufret à propos de Martin Heidegger, Paris, Aubier-Montaigne, 1983, p. 57. 
Risquons ici une analogie: Baudelaire évoque bien, dans le poème «Les phares» des Fleurs du mal (apprécié par Cézanne), Rubens, Léonard de Vinci, Rembrandt, Michel-Ange, Watteau, Goya et son contemporain Delacroix, autrement dit ce qu'il est convenu d'appeler des grands noms de la peinture, mais cela n'autorise pas pour autant à inscrire sous la rubrique de l'histoire de l'art

\section{[...] cet ardent sanglot qui roule d'âge en âge}

Il serait tout aussi inapproprié et impertinent de faire relever de l'esthétique les considérations sur l'art qui se situent hors métaphysique, hors de ses eaux territoriales. On trouve bien chez Heidegger, à partir de 1935, une pensée de l'art, voire une "philosophie de l'art», mais il n'y a pas d'esthétique de Heidegger. Les «phares» de Heidegger, quant à eux, s'appellent notamment Van Gogh, Cézanne, Paul Klee, Paula Becker-Modersohn. Sans oublier d'heureuses rencontres, dont celle avec le sculpteur Eduardo Chillida, pour nous limiter aux arts plastiques. Nous disposons à ce sujet de l'irremplaçable témoignage de l'historien de l'art Heinrich Wiegand Petzet ${ }^{4}$. Heidegger semble être allé, dans son cheminement avec la peinture, de Van Gogh à Cézanne - d'une certaine ondulation à une certaine vibration. De Chardin, Cézanne disait qu'il avait comme nul autre auparavant «nuancé l'atmosphère des choses $"$.

Encore faut-il savoir au juste ce que le mot «art» recouvre, «ce que nous appelons ainsi [was wir so nennen] ${ }^{6}$. "La plupart des hommes ont de la poésie une idée si vague que ce vague même de leur idée est pour eux la définition de la poésie», nous dit Paul Valéry dans Tel quel7. Et Heidegger comme en écho venu d'outre-Rhin: "On se fait de l'art une idée si vague que l'on tient ce vague même pour son essence» ${ }^{8}$. Ce que nous appelons ainsi - «art» - se confond en effet la plupart du temps avec «ce que l'art a été jusqu'à présent en régime métaphysique ${ }^{9}$. Selon Heidegger, comme le précise Petzet, «l'art d'aujourd'hui tout entier, qu'il soit surréaliste, abstrait ou figuratif, est d'essence métaphysique ${ }^{10}$. Ce qui

4. H. W. Petzet, Le chemin de l'étoile. Rencontres et causeries avec Heidegger, 1929-1976, C.-N. Grimbert, P. Arjakovsky (trad.), Paris, Éditions du Grand Est, 2014.

5. Conversations avec Cézanne, P. M. Doran (éd.), Paris, Macula, 1978, p. 157.

6. M. Heidegger, GA 95, 134.

7. P. Valéry, Tel quel, in Euvres, J. Hytier (éd.), Paris, Gallimard (Bibliothèque de la Pléiade), t. II, 1960, p. 547 .

8. M. Heidegger, GA 97, 296: "Man hat eine so verschwonnene Vorstellung von der Kunst, daß man meint, dieses Verschwonnene sei ihr Wesen".

9. M. Heidegger, GA 95, 135.

10. H. W. Petzet, Le chemin de l'étoile..., p. 169. 
vaut tout autant pour le national-esthétisme que fut le national-socialisme - Heidegger parle de "politische Ästhetik» ${ }^{11}$ - et les "Cahiers» abondent en remarques critiques sur l'art sous le Troisième Reich, notamment sur le wagnérisme: "Lohengrin et encore et toujours Lohengrin, les divisions blindées et escadrilles vont de pair, c'est tout un ${ }^{12}$. Le terme de «surréalisme», dont la paternité est attribuée à Apollinaire en 1917 avant que sa promotion ne soit assurée par André Breton, est interprété par Heidegger comme étonnamment proche de celui de "métaphysique ${ }^{13}$. Le surréalisme, pourrait-on dire, est métaphysique comme son nom l'indique, qui trahit crûment qu'il n'est jamais qu'une reformulation de l'intitulé "métaphysique».

Il y a donc l'art au sens de "ce que nous appelons ainsi» à tort ou à raison, dans l'idée que s'en font "la plupart des hommes", toutes époques confondues peut-être. Et il y a l'art tel qu'il se manifeste ou agonise aujourd'hui, l'art ou ce qu'il en reste - des feuilles mortes, va jusqu'à dire Heidegger:

Ce qui passe encore pour de l'«art», qu'il soit «bon» ou «mauvais», il nous faut le prendre comme autant de feuilles mortes, de feuilles dont la vie s'est retirée, privées des sucs nourriciers que l'arbre tirait de ses racines, qui virevoltent emportées par le vent et se laissent entraîner ainsi dans un «mouvement» qui leur donne un semblant de vie ${ }^{14}$.

"Autant de feuilles mortes»- de welke Blätter en lesquelles ce que l'on appelle «art» serait à présent émietté et dévitalisé. Durus hic sermo. L'expression courante en allemand pour désigner ce que nous appelons en français des feuilles mortes, y compris dans la chanson, dit littéralement que les feuilles en question sont fanées ou flétries. Il peut paraitre étrange de caractériser ce qui passe encore pour de l' «art» comme autant de feuilles mortes dans la mesure où l'on eût plutôt attendu comme antécédent «les œuvres d'art». Ou alors il faudrait dire que l'art n'est plus qu'un tas de feuilles mortes. Mais Heidegger dit bien qu'il ne nous faut prendre ce qui passe encore pour de l'art que comme autant de feuilles mortes. Comme si la décomposition ou la flétrissure ainsi diagnostiquée de l'art amenait à bouleverser aussi la syntaxe, dans un changement de construction appelé anacoluthe. Comme s'il n'y avait justement plus d'ouvres susceptibles de répondre à cette appellation. S'il n'y a plus d'œuvres d'art - à ce point

11. M. Heidegger, GA 95, 99.

12. M. Heidegger, GA 95, 133.

13. M. Heidegger, GA 97, 301.

14. M. Heidegger, GA 67, 108 ( ${ }^{\circ}$ 105: «Die Kunst»). 
disparues que Heidegger évite de recourir encore à ce terme - il n'y a plus non plus d'art. C'est pourquoi Heidegger ira jusqu'à écrire le mot «art [Kunst]» en le barrant d'une croix de Saint-André. Un art n'apparaissant plus que comme disparaissant, mais qu'il s'agit précisément de faire apparaître comme tel par cet artifice typographique, comme dés-apparaissant, desapareciendo comme on dit si bien en espagnol. Heidegger fait une croix sur l'art. L'art a vécu.

La question demeure de savoir quelle sève s'est retirée de l'art.

\section{«La » conférence sur l'origine de l'œuvre d'art}

Nous sommes en présence d'un palimpseste.

Difficulté: comment une méditation consacrée à l'art comme mise en œuvre de la vérité peut-elle elle-même se mettre en œuvre? Après le texte de «la» conférence vient une «Postface», à laquelle fait suite à son tour un «Supplément».

Nous entourons de guillemets «la» conférence en question non seulement en raison de l'existence de plusieurs versions mais encore du fait du caractère mouvant, jamais entièrement stabilisé, d'un texte dont les éclairages rétrospectifs que son auteur a pu en donner, les remaniements et apostilles qu'il a pu y apporter, ne facilitent guère la tâche, aujourd'hui, de qui se risque à en proposer une interprétation. En ne cessant de revenir sur ses tenants et aboutissants, en précisant bien des années après sur quel chemin elle se mouvait en fait, "sciemment et néanmoins tacitement $»^{15}$ - à savoir «le chemin de la question de l'essence de l'être», ce qui a pu échapper en effet à maint lecteur de bonne foi -, Heidegger aura ainsi considérablement enrichi mais aussi brouillé les pistes. Son propos semble être devenu par là insaisissable et philologiquement «indécidable». Mais cette foncière ambiguïté ${ }^{16}$ constitue aussi un atout herméneutique. Elle atteste que la méditation de Heidegger sur l'art, loin d'être verrouillée en 1935, demeure ouverte au questionnement comme au cheminement qu'elle est en elle-même, tiraillée qu'elle semble entre l'ontologie fondamentale qu'à certains égards elle prolonge, notamment dans l'approfondissement de la distinction entre Ding, Zeug et Werk, à savoir «chose», « util» et «œuvre», voire la métaphysique dont elle ne se serait pas encore entièrement déprise, et, d'autre part, la pensée de l'histoire de l'être dont plus secrètement elle

15. M. Heidegger, GA 5, 73: «wissentlich und doch unausgesprochen», où le dernier terme pourrait se traduire aussi par implicitement.

16. Ce que vise Heidegger, nous semble-t-il, en GA 67, 107, avec le terme "Zwiespältigkeit» employé à propos du texte de la conférence L'origine de l'œuvre d'art. 
procède et vers laquelle elle semble sur le point de basculer. Faut-il dès lors la reconduire à Être et temps ou bien la laisser s'en dégager?

Il existe plusieurs versions de la conférence sur L'origine de l'œuvre d'art, prononcée en 1935 et 1936 à Fribourg, Zurich puis Francfort, mais qui ne sera publiée qu'en 1950 dans les Holzwege puis dans une ultime version séparée en 1960 aux éditions Reclam, avec une postface de Hans-Georg Gadamer. La toute première version de 1935, qui n'a pas été prononcée, n'a été publiée qu'en 1989 dans le volume 5 de la revue Études heideggeriennes, l'année du centenaire de la naissance du philosophe. Mais elle diffère à ce point des versions ultérieures, tant par son contenu que par sa relative brièveté, qu'il s'agit presque d'un autre texte de même intitulé plutôt que d'une version antérieure du même texte. Elle n'est pas moins élaborée, même si elle a dormi plus d'un demi-siècle dans un tiroir, elle est autre et part parfois dans d'autres directions, comme il nous arrivera de le signaler incidemment. Le texte même de la conférence sur L'origine de l'œuvre d'art, que les Allemands appellent la Kunstwerk-Abhandlung, n'a donc pas cessé d'être remanié entre 1935 et 1960. Mais même après la parution de l'édition Reclam, Heidegger n'a pas cessé pour autant de remettre l'ouvrage sur le métier. Friedrich-Wilhelm von Herrmann, qui fut l'assistant personnel attitré de Heidegger au cours de ses quatre dernières années, nous apprend que celui-ci avait fait relier son exemplaire de l'édition Reclam en faisant intercaler par le relieur une page blanche en regard de chaque page du texte imprimé pour y consigner ces annotations que l'on appelle des apostilles ${ }^{17}$. Toutes les considérations des "Cahiers» relatives à l'art peuvent être considérées comme autant de marginalia de la conférence de 1935.

Il est toutefois permis de se demander pourquoi la publication des deux premières versions fut si longtemps différée. Petzet suggère la réponse suivante: «elles s'éloignaient par trop de l'idéologie officielle en matière d'art telle que Goebbels et Rosenberg la prêchaient comme dogme du nazisme ${ }^{18}$ - nulle part en effet il n'y est question de «race», de suprématie aryenne ou d' "art dégénéré», donc aucune concession à l'idéologie officielle en la matière, ce dont il serait temps de s'aviser. Cette «idéologie officielle» s'appelait à l'époque "Weltanschauung [vision du monde] », pour laquelle l'art était indissociable du "vécu» qu'il est censé procurer. C'est de cette «expérience vécue [Erlebnis] » que la conférence sur L'origine de l'ouvre d'art dit dans sa «Postface» qu'elle est peut-être bien «l'élément au sein duquel l'art est en train de mourir». Et d'ajouter: «Il est vrai qu'il meurt si

17. F.-W. von Herrmann, Heideggers Philosophie der Kunst: eine systematische Interpretation der Holzwege-Abhandlung, Francfort-sur-le-Main, V. Klostermann, 1980, p. XXIII (Vorwort).

18. H. W. Petzet, Le chemin de l'étoile..., p. 156. 
lentement qu'il lui faut, pour mourir, quelques siècles ${ }^{19}$. Francesco Alfieri a relevé le nombre impressionnant d'occurrences du terme Erlebnis dans les Apports à la philosophie ${ }^{20}$. Il n'est pas possible d'en faire abstraction lorsque c'est la question de l'art à l'époque des Temps nouveaux qui est en jeu.

La conférence sur L'origine de l'ouvre d'art est donc strictement contemporaine de la mise en chantier des grands traités historiaux, à commencer par les Apports à la philosophie de 1936-1938. Elle ouvre la voie à toutes les considérations ultérieures de Heidegger sur l'art, y compris dans les "Cahiers». En d'autres termes, le questionnement heideggerien sur l'art comme «mise en œuvre de la vérité» est indissociable du fil rouge que constitue la question en quête de la vérité de l'être.

C’est là ce que précise rétrospectivement le «Supplément» ajouté par Heidegger en 1960 (mais rédigé en 1956) pour l'édition Reclam: «Tout l'essai sur L'origine de l'œuvre d'art se meut sciemment, et néanmoins tacitement, sur le chemin de la question de l'essence de l'être. La méditation sur ce qu'est l'art est entièrement et décisivement déterminée par la seule question de l'être» ${ }^{21}$. "La seule question de l'être» telle qu'elle n'est plus abordée alors dans la perspective de l'ontologie fondamentale élaborée en 1927 par Être et temps mais dans celle de l'histoire de l'être, de la « vérité de l'être» dont témoigne le grand art où elle vient irradier. L'essai de Heidegger ne vise pas à fournir une réponse définitive ni même tranchée à la question "qu'est-ce que l'art? » mais à donner quelques aperçus essentiels quant à ce dont il retourne avec l'œuvre d'art en se laissant interpeller par l'art lui-même en ses œuvres quitte à laisser intacte l'énigme que l'art est à lui-même.

On y trouve notamment, comme chacun sait, des développements consacrés à un tableau de Van Gogh, Les souliers (sans doute s'agit-il de Schoenen, 1886, Amsterdam, musée Van Gogh ${ }^{22}$ ) - développements euxmêmes fameux et peut-être un peu trop célèbres ne serait-ce que par la place qu'ils occupent dans la littérature heideggerienne où ils jouent un

19. M. Heidegger, GA 5, 67; trad. fr.: Chemins qui ne mènent nulle part, trad. de W. Brokmeier revue et corrigée avec le concours de J. Beaufret, F. Fédier et F. Vezin, Paris, Gallimard (Idées), 1980, p. 90.

20. F.-W. von Herrmann, F. Alfieri, Martin Heidegger. La verità..., p. 148-151 (note 14); p. 112-114 (note 13) de la version allemande (Martin Heidegger. Die Wahrheit über die "Schwarzen Hefte", Berlin, Duncker \& Humblot, 2017).

21. M. Heidegger, GA 5, 73; Chemins..., p. 97 (nous soulignons).

22. Sur le problème de l'identification du tableau de Van Gogh auquel se réfère Heidegger, comme sur les errements positivistes auxquels il a pu donner lieu ("presque comme une caricature du travail d'historien de l'art»), nous invitons le lecteur à se reporter à la notice de H. France-Lanord sur Schapiro dans le Dictionnaire Martin Heidegger, P. Arjakovsky, F. Fédier, H. France-Lanord (dir.), Paris, Cerf, 2013, p. 1187-1189. 
rôle analogue à celui de la toile Guernica de Picasso aux yeux d'un certain public peu averti des choses de la peinture, instrumentalisée en "message» qu'elle serait censée "véhiculer». La peinture est dès lors réduite à une imagerie, que celle-ci illustre l'attachement à la glèbe, l'âpreté et l'humilité de la vie paysanne, ou «symbolise» les horreurs de la guerre à travers une "dénonciation» des crimes commis en 1937 en Biscaye par le nazisme et le franquisme réunis. Le taureau, le cheval? Symboles, allégories. Au fond, Guernica serait mutatis mutandis une réédition du tableau non moins fameux de Goya El tres de mayo. Il suffirait pour s'y retrouver de remplacer la brutalité de la soldatesque napoléonienne par celle de la légion Condor. Mais pour se retrouver où au juste? "Là où nous avons coutume d'être", conformément à nos «bonnes vieilles habitudes».

La grandeur du «grand public» et celle du «grand art» sont des grandeurs qui s'annulent mutuellement. La toute première version de la conférence sur L'origine de l'œuvre d'art allait même jusqu'à affirmer sans ménagements que «la grandeur d'une œuvre d'art se mesure à sa force de destruction [Zerstörungskraft]» du public ${ }^{23}$, pour autant que celui-ci ne peut ressortir indemne de l'épreuve à laquelle le confronte le grand art. L'art cesse d'être fidèle à sa vocation dès lors qu'il vise à répondre à la demande d'un public, multitude ou petit cénacle.

Si saisissante soit-elle, la description phénoménologique que donne Heidegger d'un tableau de Van Gogh, véritable leçon de choses, ne prend son sens que située dans l'économie du propos de la conférence sur L'origine de l'œuvre d'art, et non isolée comme un morceau de bravoure. Suite aux développements qu'il consacre au tableau de Van Gogh, Heidegger dit quant à lui sobrement: "Nous n'avons rien fait que nous mettre en présence du tableau de Van Gogh. C’est lui qui a parlé [Dieses hat gesprochen]. La proximité de l'œuvre nous a soudain transporté ailleurs que là où nous avons coutume d'être ${ }^{24}$. C'est lui, le tableau, qui a parlé. Si l'œil écoute, comme le dit Paul Claudel, c'est bien que le tableau parle. De même qu'on ne dit pas d'un tuyau d'orgue qu'il chante mais qu'il parle - il suffit d'écouter de grands compositeurs pour orgue tels que Buxtehude, Vivaldi ou Bach pour l'entendre. "Ailleurs que là où nous avons coutume d'être » et cependant là où nous sommes proprement si nous passons des visites aux visitations: si, loin de nous contenter de quelques visites dans différents musées et de la fréquentation de salles de concert, nous nous laissons visiter par les œuvres d'art en ce qu'elles peuvent avoir de bouleversant et de renversant et

23. M. Heidegger, «Vom Ursprung des Kunstwerkes: Erste Ausarbeitung», Études heideggeriennes, vol. 5,1989 , p. 8 .

24. M. Heidegger, GA 5, 21; Chemins..., p. 36. 
œuvrons nous-mêmes par là à préparer la métamorphose de l'être humain en Dasein propre à inaugurer un «autre commencement». La visitation, c'est ce que Heidegger appelle sobrement: se laisser dire quelque chose par l'œuvre d'art.

Là où le tableau nous a soudain transportés, littéralement ravis, là s'éveille en l'homme le Dasein.

Être transporté, «ravi» ailleurs que là où nous avons coutume d'être, c'est ce qui est arrivé à Dostoïevski à Bâle devant le tableau de Holbein le Jeune Le Christ mort [Der tote Christus im Grabe].

La référence de Heidegger à un tableau de Van Gogh résulte d'une rencontre entre le penseur et le peintre néerlandais qui semble remonter à l'année 1931 si l'on en juge d'après le témoignage qu'en donne une lettre adressée alors par Heidegger à son épouse Elfride:

J'ai passé toute une matinée en compagnie des Van Gogh. [...] Les tableaux m’ont vivement impressionné, et même profondément remuée ${ }^{25}$.

En 1922, Heidegger écrivait à Karl Jaspers n'avoir encore jamais vu une toile originale de Van Gogh (lettre du 27 juin 1922). Il en avait lu les lettres, comme le montre un passage du cours du semestre d'été 1923, Ontologie. Hermeneutik der Faktizität, où Van Gogh est décrit comme un écorché vif s'étant "pour ainsi dire arraché les toiles du corps ${ }^{26}$. Il revient incidemment au peintre néerlandais dans le cours du semestre d'hiver 1935-1936, Die Frage nach dem Ding [Qu'est-ce qu'une chose?], où il évoque "cette chaise rustique avec la pipe, que montre la peinture de Van Gogh ${ }^{27}$. Il y revient encore dès l'année suivante dans le cours du semestre d'hiver 1937-1938 intitulé Grundfragen der Philosophie, où le nom de Van Gogh apparaît en compagnie de ceux de Schiller, Hölderlin, Kierkegaard et Nietzsche ${ }^{28}$. Visiblement, la peinture de Van Gogh le hantait.

25. M. Heidegger, lettre du 17. VIII. 1931, in «Mein liebes Seelchen!». Briefe Martin Heideggers an seine Frau Elfride: 1915-1970, G. Heidegger (éd.), Munich, Deutsche Verlags-Anstalt (DVA), 2005, p. 270. Toutefois, d'après Meyer Schapiro, c'est en mars 1930 que Heidegger aurait vu pour la première fois le tableau de Van Gogh en question à Amsterdam. Comme il l'écrit dans sa note intitulée The Still Life as a Personal Object: A Note on Heidegger and Van Gogh (in The Art of Art History: A Critical Anthology, D. Preziosi (éd.), Oxford, Oxford University Press, 2009, p. 297): "In reply to my question, Professor Heidegger has kindly written me that the picture to which he referred is one that he saw in a show at Amsterdam in March 1930. This is clearly de la Faille's no. 255 ; there was also exhibited at the same time a painting with three pairs of shoes, and it is possible that the exposed sole of a shoe in this picture, inspired the reference to the sole in the philosopher's account».

26. M. Heidegger, GA 63, 32.

27. M. Heidegger, GA 41, 213.

28. M. Heidegger, GA 45, 216. 


\section{L'art comme mise en œuvre de la vérité}

La détermination de l'art comme "mise en œuvre de la vérité» est toutefois loin d'aller de soi. Elle n'a rien d'évident. Elle est d'autant moins évidente que la locution «mise en œuvre» est devenue à ce point monnaie courante qu'elle finit par passer entièrement inaperçue. Mais il y a mettre en œuvre et mettre en œuvre. «Mettre en œuvre», c'est réaliser ou mettre en chantier un projet déjà arrêté qu'il ne s'agirait plus, comme on dit aujourd'hui, que de «finaliser ». "Mettre en œuvre», c'est appliquer, exécuter, réaliser. Lorsque la marquise de Sévigné écrit à la comtesse de Grignan, sa fille: «Je souhaite de mettre en œuvre les résolutions que j'ai prises sur mes réflexions» (lettre du 14 juillet 1680); il s'agit de les mettre en pratique, d'agir de telle sorte que lesdites résolutions ne demeurent point lettre morte, qu'elles ne restent justement pas au stade de «bonnes résolutions». Il lui faut, comme l'écrivait Descartes quelque trente années auparavant dans l'article 153 de son traité Les passions de l'âme, "ne manquer jamais de volonté pour entreprendre et exécuter toutes les choses qu' [elle] jugera être les meilleures».

Tout autre est la «mise en œuvre» dont parle Heidegger. C'est pourquoi l'expression "Ins-Werk-setzen», rendue par «mise en œuvre», doit être entendue dans toute sa plénitude et pour ce faire arrachée à sa platitude. L'expression ins Werk setzen est bien attestée depuis le moyen haut allemand, parmi d'autres tournures usuelles équivalentes, comme l'atteste le dictionnaire des frères Grimm (Deutsches Wörterbuch) à l'article «Werk»:

etwas gedachtes, geplantes, verheiszenes u. dgl. ins (zu) werk bringen, führen, richten, setzen, stellen oder ziehen: in eine Tat umsetzen, zur Ausführung bringen (vgl. bewerkstelligen)

Parmi toutes ces tournures usitées, Heidegger en retiendra deux, que nous faisons figurer en gras ci-dessus: ins Werk setzen et ins Werk stellen. Pour le sens, notre tournure équivaut donc à «mettre à exécution [zur Ausführung bringen]", ou encore, de manière ramassée, bewerkstelligen, verbe plus «fonctionnel» apparu au XVII ${ }^{e}$ siècle. Tel est donc le sens courant de cette locution ins Werk stellen, centrale dans la conférence sur L'origine de l'ouvre d'art, mais seulement le sens courant et fatigué par l'usage. Ce sens courant fait de la tournure ins Werk setzen l'exact équivalent de bewerkstelligen, à savoir la «mise en œuvre» dans le langage fonctionnel, opérationnel d'aujourd'hui comme accomplissement d'un programme, par exemple tout mettre en œuvre pour empêcher la guerre, ou encore concrétiser un projet, réaliser des travaux. Il est à noter qu'une telle mise en œuvre n'a précisément plus aucun rapport avec quoi que ce soit qui ressortisse à une œuvre proprement dite, à ce que les musicologues appellent encore 
un opus dans la numérotation des œuvres d'un compositeur. On connait la célèbre réplique de Michel-Ange au pape Jules II : non possum descendere, magnum opus facio $^{29}$. C'est qu'il y a dans l'occupation du peintre ce que Maurice Merleau-Ponty appellera, au début de L'oil et l'esprit, « une urgence qui passe toute autre urgence ${ }^{30}$. Le timing des opérations, fussent-elles pontificales, ne saurait prévaloir sur le tempo de l'œuvre. Heidegger, quant à lui, nous invite à réentendre l'expression ins Werk setzen qu'il substantive à partir de ce que lui dit sa langue: non pas simplement mettre à exécution, mettre en œuvre, mais établir ou instituer en une œuvre digne de ce nom, et qui d'elle-même s'impose comme telle, configurer à la mesure d'une œuvre. Configurer à la mesure d'une «œuvre», «de ce qui jadis s'appelait ainsi et - historicisme oblige - en a gardé le nom ${ }^{31}$.

Setzen est en allemand le causatif de sitzen, "être assis», c'est-à-dire avoir trouvé une assise. Mettre en œuvre la vérité, c'est donc faire en sorte que celle-ci trouve une assise qui lui siée. Apparenté aux verbes sitzen et setzen est le verbe grec ह̌ $\zeta$ o $\mu a \mathrm{l}$, «être assis », ainsi qu'en grec ancien comme moderne $\varepsilon \delta \rho \alpha$, "siège ", que nous retrouvons dans cathèdre et cathédrale, puis chaire et finalement chaise, mais encore i $\delta \rho \nu \mu \alpha$, «ce qui est fondé » ou institué, notamment un temple ou une statue. Après la description phénoménologique d'un tableau de Van Gogh, c'est à point nommé que Heidegger en vient à illustrer son propos en se référant à un temple grec. En tant qu'í $\delta \rho v \mu \alpha$, le temple grec est par excellence ce qui est fondé ou institué comme attestation humaine de la présence du divin. D’où le développement que Heidegger consacrera à l'instauration en recourant au verbe si hölderlinien stiften, au triple sens de l'offrande, de la fondation et de l'inauguration - schenken, gründen, anfangen ${ }^{32}$. Heidegger explicite le verbe setzen, dans la locution "Ins-Werk-setzen", par le verbe stellen, "poser», "installer», autrement dit faire venir à stance. Or, les verbes apparentés sitzen et setzen représentent un élargissement de la racine indo-européenne *es- dont provient également le verbe irrégulier sein en allemand [être] à la troisième personne de l'indicatif présent: ist. Dans le deuxième chapitre de son cours de 1935 Introduction en la métaphysique, Heidegger recense les trois racines indo-européennes auxquelles remontent les flexions du verbe «être» en allemand, sein. En ce qui concerne la première il dit ceci:

29. Il s'agit en fait d'une citation de la Bible en 2 Esdras (= Néhémie) 6, 3. Vulgate: Opus grande facio, et non possum descendere, ce qui, selon Proust, «devrait être la devise de tout artiste» (À la recherche du temps perdu, J.-Y. Tadié (éd.), Paris, Gallimard (Bibliothèque de la Pléiade), t. IV, 1998, p. 838).

30. M. Merleau-Ponty, L'œil et l'esprit, Paris, Gallimard, 1964, p. 15.

31. M. Heidegger, GA 95, 135.

32. M. Heidegger, GA 5, 63 sq.; Chemins..., p. 84 sq. 
La racine la plus ancienne, l'étymon proprement dit est *es-, en sanscrit asus, la vie, le vivant, ce qui trouve en soi-même à partir de soi-même sa propre tenue, ce qui va et repose à partir de soi-même: ce à quoi il ne tient qu'à soi de se tenir ${ }^{33}$.

Ce qui est mis en œuvre trouve ainsi à se tenir à partir de soi-même, et par là à être.

Mais la vérité ainsi mise en œuvre n'est pas, quant à elle, la vérité traditionnellement conçue comme justesse ou exactitude dans l'adéquation de la pensée et de la chose. Elle est entendue comme Unverborgenheit, présence à découvert, hors retrait, désoccultation ou déclosion, comme il ressort du $₫ 44$ d'Être et temps: ả $\lambda \dot{\theta} \theta \varepsilon \iota$.

La thèse heideggerienne centrale selon laquelle l'art est mise en œuvre de la vérité - de la vérité de l'étant en son être - n'aurait pu être formulée sans cet acquis majeur d'Etre et temps dont elle est indissociable: la dimension de l'à $\lambda \dot{\theta} \theta \varepsilon \iota a$.

Au mot allemand Kunst répond le grec $\tau \dot{\varepsilon} \chi \nu \eta$ que les Latins ont traduit par ars, aussi bien au sens de l'artisan que de l'artiste, du bon ouvrier que du virtuose, des arts et métiers que des beaux-arts: là où s'exerce un savoir qui demande du métier ${ }^{34}$. Alors y aurait-il simple passage de témoin, sans solution de continuité, entre le monde grec et le monde romain, entre la statuaire grecque archaïque ou classique et les «copies romaines d'époque hellénistique», comme disent les musées? Ou bien, plus profondément, une mutation radicale? Faut-il aller jusqu'à dire que l'artisan grec est un artiste et l'artiste romain un artisan? C'est ce que suggère Jean Beaufret, en écrivant plus subtilement à propos du monde grec: «ses artisans sont plus près des artistes là où, dans la vision romaine des choses, les artistes eux-mêmes ne sont plus que des agents ${ }^{35}$. Entre le monde grec et le monde romain s'est opéré un renversement de perspective.

Toujours est-il que la $\tau \dot{\varepsilon} \chi \nu \eta$, avant de devenir ars, est étroitement mise en rapport par Aristote, dans l'Éthique à Nicomaque (VI, 3), avec l'à $\lambda \dot{\theta} \theta \varepsilon ı$. En voyant dans l'art la mise en œuvre de la vérité, Heidegger semble donc au plus près d'Aristote, qu'à vrai dire il approfondit plus qu'il ne le reprend.

33. M. Heidegger, GA 40, 75; En guise de contribution à la grammaire et à l'étymologie du mot "être", P. David (éd. et trad.), Paris, Seuil, 2005, p. 61.

34. Voir F. Vezin, «La question de l'œuvre d'art», in La fête de la pensée. Hommage à François Fédier, H. France-Lanord, F. Midal (dir.), Paris, Lettrage, 2001, p. 259-273.

35. J. Beaufret, De l'existentialisme à Heidegger, Paris, J. Vrin, 1986, p. 70. Cette citation est extraite d'une longue note que Jean Beaufret avait intercalée dans son propre exemplaire aux pages 102-103 de l'édition originale de 1971 (parue aux éditions Denoël sous le titre Introduction aux philosophies de l'existence) comme le signale Guy Basset dans sa note liminaire pour l'édition de 1986. 
Car l'ả $\lambda \dot{\theta} \theta \varepsilon ı$, si elle a bien été nommée et éprouvée par les Grecs, n’a pas été pour autant pensée comme telle. C'est pourquoi il ne s'agit pas seulement de revenir aux Grecs mais de tenter de dégager ce qui demeure implicite

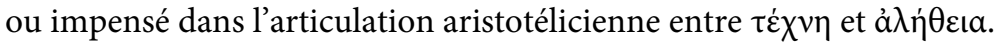

La conférence sur L'origine de l'œuvre d'art n'aurait sans doute pas eu lieu d'être, privée de cet élément au sein duquel elle se déploie: l'à $\lambda \dot{\eta} \theta \varepsilon \imath$, l'Unverborgenheit, la vérité entendue comme dévoilement. L'art est mise en œuvre de la vérité comme déclosion. Il est ainsi une manière insigne dont advient la vérité. C'est sur cette lancée que Heidegger peut dire dans les Überlegungen VIII que «l'“œuvre” [Werk] est répercussion [Wirkung] "de" l'être ${ }^{36}$. Puis Heidegger entoure de guillemets le terme «Wirkung", qui dans le vocabulaire de la métaphysique désigne l'effet dans la relation de cause à effet, comme dans la seconde analogie de l'expérience dégagée et analysée par Kant dans la Critique de la raison pure. Wirkung ne doit pas être entendu ici comme renvoyant à un problème de causalité, comme l'effet d'une cause, mais à partir de Werk, l'œuvre. Celle-ci est répercussion de l'être, bien plutôt que l'effet d'une cause efficiente qui serait en l'occurrence l'activité créatrice de l'artiste. Dans la perspective qui est celle de l'histoire de l'être, l'œuvre est œuvrée par l'être dont le resplendissement en elle s'appelle le beau. Le beau est le vrai dans toute sa splendeur, celle de son éclosion dans l'étant comme une lumière venue d'ailleurs. L'être qui brille par son absence en tout étant brille par sa présence dans l'œuvre d'art comme éclosion de l'étant dans son être. Ou disons plus prudemment: l'œuvre d'art est un étant où l'être ne brille pas seulement par son absence. C'est pourquoi la question de la provenance essentielle de l'œuvre d'art ne relève pas du strict domaine de l'esthétique, c'est une question ontologique ${ }^{37}$. Mais encore et surtout: en originant l'œuvre dans l'être comme sa répercussion, les «Cahiers» accentuent délibérément la provenance de l'œuvre à partir de l'histoire de l'être et détachent la notion même d'œuvre de ce qu'elle pouvait

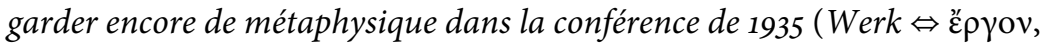
$\varepsilon \dot{v} \varepsilon \dot{\rho} \gamma \varepsilon ı$ selon les déterminations de «jadis $»^{38}$ ) en la rattachant à l'histoire de l'être comme son véritable foyer.

La vérité de l'œuvre n'est pas telle qu'en celle-ci la vérité se trouverait figée, enfin fixée et inamovible, comme le tableau dans son cadre accroché au mur ou la statue sur son socle. La vérité de l'œuvre ne réside dans l'œuvre

36. M. Heidegger, GA 95, 137.

37. F.-W. von Herrmann, Heideggers Philosophie der Kunst..., p. 60.

38. M. Heidegger, GA 5, 69 (Chemins..., p. 92): damals. Cette dernière page de la «Postface» à la conférence sur L'origine de l'œuvre d'art nous livre un raccourci saisissant de l'ensemble des principales déterminations du beau dans l'histoire de la métaphysique depuis Platon et Aristote. 
que pour autant qu'elle est et ne cesse d'être im Werk am Werk, «à l'œuvre dans l'œuvre » ${ }^{39}$, c'est-à-dire ne cesse d'y advenir ou d'y être en gestation [geschehen]. C'est là une percée vers l'Ereignis, cette fulgurante avenance dont le nom ne figure pas dans la conférence de 1935, mais seulement dans son «Supplément» de $1956^{40}$.

Ce qui, dans l'œuvre, est proprement à l'œuvre n'est autre que l'avènement de la vérité comme ouverture de l'étant dans son être.

Nous sommes à présent en mesure de mieux discerner l'apport des «Cahiers de travail» à la thématisation de l'art dans le cheminement de la pensée de Heidegger: leurs incursions apparemment décousues dans ce domaine développent les apostilles nécessairement limitées et "économiques» ajoutées par Heidegger sur son exemplaire de la conférence sur L'origine de l'ouvre d'art.

\section{D’un art consommé à la consomption de l'art}

On appelle art consommé un art qui a atteint un haut degré de perfection, quand l'artiste a fait ses preuves. On peut comprendre à partir de là pourquoi ce que nous appelons art se dit en russe иску́сстьо, du latin experimentum, du fait de l'expertise dont il consiste à faire preuve. Mais si la consommation consiste à amener une chose à son plein accomplissement, elle peut désigner aussi l'action ayant pour effet de la détruire. Il n'y a pas loin de la consommation à la consomption. C'est là toute l'ambiguïté qui demande à être pensée dans le double sens du terme "Vollendung» employé par Heidegger: à la fois accomplissement et achèvement.

Dans la mesure où l'art a cessé de répondre à cette vocation, mettre en œuvre la vérité de l'être - et c'est ici la question de la fin de l'art que Heidegger aborde et reprend à nouveaux frais -, il déserte le site qui est le sien et se trouve dès lors comme désaffecté, comme si les œuvres elles-mêmes se trouvaient en quelque sorte désœuvrées par l'oubli de l'être autant qu'elles ont pu être œuvrées par l'être. C'est le phénomène de cette désaffection de l'être et désaffectation concomitante de l'art que vise Heidegger en parlant de la fin de l'art qui n'en finit pas de finir.

Cette caractérisation pourrait paraître négative comme dépréciation de l'art du XX $\mathrm{XX}^{\mathrm{e}}$ siècle. Elle ne l'est pas dans la mesure où l'oubli de l'être appartient lui-même à l'histoire de l'être comme l'éclipse du Soleil à sa carrière. Certes, Heidegger a pu déclarer aux journalistes du Spiegel, en 1966,

39. M. Heidegger, GA 5, 21; Chemins..., p. 36.

40. M. Heidegger, GA 5, 73; Chemins..., p. 97. 
que «la littérature d'aujourd'hui, par exemple, est largement destruktiv» ${ }^{41}$. On aurait aimé quelques précisions, que les interlocuteurs d'alors n'ont malheureusement pas demandées. Faut-il comprendre la littérature allemande d'après-guerre dans son ensemble? La littérature européenne? Mondiale? Mais depuis quand? Des noms! Toujours est-il que l'adjectif destruktiv n'est pas à entendre ici au sens de la Destruktion entendue par Heidegger comme dés-obstruction de la tradition. Dans son contexte, celui du déracinement de l'homme qui va de pair avec le règne du fonctionnement technique de toutes choses, Heidegger vise ici un trait qu'il perçoit dans «la littérature d'aujourd'hui», à savoir sa participation à ce déracinement dans la liquidation de tout héritage, une démolition en règle de tout ce qui avait pu constituer un ancrage et un enracinement dans une tradition. C'est pourquoi des écrivains tels qu'Adalbert Stifter et Knut Hamsun lui semblent appartenir à un temps révolu, où la littérature n'était pas destruktiv.

Mais l'art est devenu l'affaire de la "politique culturelle» en son affairement, il entre désormais dans le circuit du Kunstbetrieb qui lui assigne un «rôle à jouer» et une «valeur». Il tourne ainsi à plein régime métaphysique en délaissant ou en ignorant la possibilité qu'il tient en réserve d' «un art qui serait autre» que celui porté par la métaphysique en préparant la métamorphose, en l'être humain, du sujet en Dasein; d'un art qui trancherait et serait ainsi une véritable dé-cision, une Ent-scheidung, et par là aussi une césure. En d'autres termes, l'apparente sévérité du jugement porté par Heidegger sur la littérature de son temps est l'envers d'une attente: celle d'un art faisant incursion dans l'autre commencement, qui tranche entre le premier commencement et l'autre commencement - ce que n'ont pas su faire, à ses yeux, l'art abstrait, le surréalisme et le suprématisme (l'art gegenstandslos ou «sans objet» de Malévitch) ni aucune «avant-garde»-, et soit porteur, inauguralement, d'une histoire. Telle est bien la haute idée que Heidegger se fait de l'art comme haut lieu de la vérité que l'art de son temps lui semble avoir en grande partie désertée - d'autant plus attentive sera son écoute de poètes de l'envergure de Paul Celan et René Char.

L'art n'est pas envisagé par Heidegger dans une perspective historique mais historiale, en tant qu'il est porteur d'histoire et susceptible de laisser advenir, à partir de lui, la possibilité que s'amorce une histoire autre, celle précisément de l' "autre commencement». On peut relever à cet égard une remarque incidente de la toute première version du texte de la conférence sur L'origine de l'œuvre d'art, mais qui ne sera pas reprise dans les versions

41. M. Heidegger, GA 16, 670; trad. fr.: Écrits politiques, 1933-1966, F. Fédier (éd. et trad.), Paris, Gallimard, 1995, p. 259. 
ultérieures: «il n'y a pas d'art préhistorique, car avec l'art c'est d'ores et déjà une histoire qui a commencé» ${ }^{42}$.

Mais au stade ultime de l'accomplissement et achèvement [Vollendung] du règne de la métaphysique qu'est la faisance [Machenschaft] généralisée, l'art ne peut plus nous être qu'objet de délectation au sein de l'expérience vécue [Erlebnis] : l'œuvre elle-même disparaît derrière «ce que cela nous fait». À l'univers de la facture s'est substitué celui de la faisance. L'homme réduit à lui-même - plutôt que pensé à partir de son ouverture à l'être tourne dès lors en rond dans la cage dorée de sa subjectivité. C'est le triomphe du kitsch, de l'art qui assume comme dimension artistique ce qui est purement artificiel ${ }^{43}$, et par là de l'artefact et du produit sur l'œuvre. "Nous avons des produits, nous n'avons plus d'œuvres», dit Balzac en 1839 au début de Béatrix, en profond visionnaire qu'il fut. Cette phrase de Balzac pourrait être mise en exergue de la méditation de Heidegger sur l'art de notre temps.

Mais il reste à savoir en quel(s) sens l'art d'aujourd'hui peut être qualifié de métaphysique et trouve avec l'achèvement de la métaphysique son propre achèvement et sa consomption. On peut relever quatre sens distincts mais solidaires entre eux:

1. dans la mesure où l'art lui-même se comprend comme relevant de l'esthétique, donc du sensible et par là de la distinction platonicienne institutrice de la métaphysique qui oppose le sensible et l'intelligible;

2. dans la mesure où il donne lieu, à partir de là, à l'esthétisme;

3. pour autant que Machenschaft et Erlebnis, faisance et expérience vécue, déterminent de fond en comble et saturent notre vision de l'art et notre manière de nous y rapporter;

4. pour autant que ces deux déterminations s'inscrivent à leur tour dans le cadre de la métaphysique de la subjectivité, asphyxiant toute possibilité de rapport à des œuvres dans ce que les «Cahiers» appellent de façon récurrente "Vermenschung des Menschen", ce que nous proposons de rendre par «réduction de l'homme à lui-même».

Les trois derniers sens relèvent des Temps nouveaux. Ils appellent nombre de développements dans les «Cahiers", ce qui nous permet d'affirmer que la place qu'y occupe la question de l'art excède largement la place

42. M. Heidegger, «Vom Ursprung des Kunstwerkes: Erste Ausarbeitung», p. 21: «gibt es keine vorgeschichtliche Kunst, weil mit der Kunst schon Geschichte angefangen hat".

43. M. Heidegger, GA 66, 31. 
qu'on voudra lui accorder sur la base du nombre de passages où celle-ci se trouve expressément thématisée.

L'esthétisme est sous le Troisième Reich l'instrumentalisation de l'art dans l'organisation du vécu par la propagande officielle diffusée au nom d'une "politique culturelle [Kulturpolitik] » à destination des masses et dite alors «volksgebunden», «étroitement liée au peuple», "proche des gens». Cette politique passe notamment par le monumental ou colossal (figures du «gigantesque» et du gigantisme comme promotion du quantitatif en qualitatif: plus, c'est mieux), le culte et l'exaltation du corps sain, la jouissance calculée - il n'est que de songer ici à Albert Speer, Arno Brecker, Leni Riefenstahl, Karl Orff et Wilhelm Reich. Ce que résume aux yeux de Heidegger le wagnérisme: «Wagner avait besoin de wagnériens et de wagnériennes », dit un cours de Heidegger sur Nietzsche ${ }^{44}$. D'où le rapprochement déjà évoqué entre Lohengrin à satiété et les divisions blindées: le tout est de revenir à la charge, avec pour dénominateur commun la force de frappe. Nulle part sans doute Heidegger n'aura autant parlé de Wagner que dans ses cours sur Nietzsche et dans les «Cahiers». Dans «le triomphe de Richard Wagner au XX ${ }^{\mathrm{e}}$ siècle ${ }^{45}$ il voit une attestation de la fin de l'art. Non seulement la musique de Wagner vise à subjuguer ses auditeurs, mais elle devient l'art par excellence, qui rassemble en lui et autour de lui tous les arts ${ }^{46}$ en tant qu' «œuvre d'art totale [Gesamtkunstwerk]», elle se voit promue en genre artistique déterminant ${ }^{47}$. Cette promotion de la musique assurée par Nietzsche et Wagner repose selon Heidegger sur la récupération de la calculabilité qui lui est inhérente mise au service de la consécration de l'expérience vécue, de la délectation dans le «ressenti». C'est la consécration de l'affect de masses subjuguées et manipulées qui entrent en transe, l'aspect orgiaque du nazisme qui ne se limite pas à la SA (Sturmabteilung) éliminée le 30 juin 1934 lors de la Nuit des longs couteaux ou, en termes nietzschéens, du dionysiaque qui, n'étant plus tempéré ou contrebalancé par l'apollinien cesse aussitôt d'être dionysiaque dans l'absence criante de forme [Formlosigkeit ${ }^{48}$ ], la griserie [Rausch] et la brutalité ${ }^{49}$. Le terme Rausch est apparenté à l'anglais rush: la ruée vers tout ce qui donne le vertige. Ce Rausch que devient l'expérience vécue en se laissant emporter

44. M. Heidegger, GA 43, 104.

45. M. Heidegger, GA 95, 52.

46. M. Heidegger, GA 95, 132.

47. M. Heidegger, GA 95, 150: «maßgebende Kunstgattung».

48. M. Heidegger, GA 43, 139.

49. Sur la question très importante de la «brutalité» dans les «Cahiers", nous renvoyons à notre article «La brutalité de l'animal comptable. Pour accéder aux Cahiers noirs», Études heideggeriennes, vol. 32, 2016, p. 91-104. 
par son propre élan, nul ne l'a sans doute mieux caractérisé qu'Alfred de Musset dans un vers passé en proverbe de «La coupe et les lèvres»:

Qu'importe le flacon pourvu qu'on ait l'ivresse?

- où ivresse rime avec maîtresse. Qu'importe l'art, en effet, s'il est rabaissé à un fournisseur d'expériences vécues... C'est ce «qu'importe» qui signale avec éloquence le phénomène que Heidegger appelle la fin ou l'achèvement de l'art. Mais c'est toute la page 150 du tome 95 de l'édition intégrale (Überlegungen VIII, $\mathrm{n}^{\circ} 33$ ), qui date de 1938 , qu'il faudrait pouvoir citer ici, à laquelle nous nous contenterons de renvoyer le lecteur.

Si le commerce de l'art fait ses choux gras du commerce d'œuvres d'art devenues autant de marchandises ayant une cotation à l'instar de valeurs boursières, l'expérience vécue elle aussi se goberge, qui en fait ses délices. Délices qu'il ne s'agit que de rendre accessibles au plus grand nombre - d'où de nos jours le "pass culture pour les jeunes» après la «Fête de la musique». Cela s'appelle la «démocratisation de la culture» reposant sur le «budget de la culture» dans le cadre d'une "politique culturelle» - cette Kulturpolitik au sujet de laquelle Heidegger souligne dans les "Cahiers» qu' «il revient aux Français d'avoir inventé cette création bien étrange au demeurant $~^{50}$. Il n'y a pas aujourd'hui d'institution digne de ce nom qui n'ait sa «mission culturelle». Loin de la cultura évoquée dans «Bâtir, habiter, penser ${ }^{51}$, la promotion de la culture est ainsi devenue ce que Heidegger va jusqu'à qualifier de "culture de la culture ${ }^{52}$.

L'expérience vécue est donc délectation. Jean Beaufret nous dit à ce sujet:

Le terme est révélateur. Délectation paraît être de la même famille que lecture, et se rattacher par là à ce que les Grecs avaient nommé logos. Mais ce n'est là qu'apparence. Le mot provient en réalité d'une tout autre racine qui dit que celui qui se délecte est tout simplement alléché par l'appât d'une friandise. Heidegger écrit dans Introduction à la métaphysique: «Pour les Grecs, être et beau étaient synonymes. Aujourd'hui, le beau relève du domaine du confiseur ». Bien sûr, la sucrerie esthétique a besoin d'être relevée d'épices ou de piments divers. Mais enfin, c'est toujours la même marchandise ${ }^{53}$.

50. M. Heidegger, GA 95, 323. Voir à ce sujet la notice d'A. Froidecourt intitulée «Politique culturelle» dans le Dictionnaire Martin Heidegger, p. 1064-1065.

51. M. Heidegger, GA 7, 149.

52. M. Heidegger, GA 95, 13: «Kultur-Kultur». Quant à voir là une quelconque hostilité de Heidegger vis-à-vis de la culture, nous en laissons bien volontiers le soin aux obscurantistes de service.

53. J. Beaufret dans: E. de Rubercy, D. Le Buhan, Douze questions..., p. 57-58. La citation de Heidegger est tirée de GA 40, 140; trad. fr.: Introduction à la métaphysique, G. Kahn (trad.), Paris, Gallimard, 1967, p. 140. 
Comprenons que cette marchandise est frelatée. Du beau artistique elle ne retient que ce qu'il peut avoir d'attrayant, que Baudelaire appelait pour sa part «l'enveloppe amusante, titillante, apéritive du divin gâteau » 54 .

La délectation est un autre nom de la jouissance artistique, qui savoure les œuvres d'art et s'en régale. Le goût est ramené au plaisir gustatif, tel qu'il relève selon Kant non du beau mais de l'agréable. Mais elle a aussi, cette délectation, ses lettres de noblesse philosophiques et même théologiques. La définition communément acceptée du beau à l'époque médiévale est «id quod visum placet»: ce qui plaît quand on le voit, ce à la vue de quoi nous éprouvons du plaisir, la vue ayant aussi ici cette fonction vicariante soulignée par saint Augustin dans ses Confessions (X, 35). Le plaisir éprouvé en présence du beau est tenu pour constitutif de ce qu'il est. Nicolas Poussin dira de la peinture: "sa fin est la délectation ${ }^{55}$. Descartes ne commence pas autrement son Compendium musicae ou Abrégé de musique lorsqu'il dit d'entrée de jeu, au sujet de la musique: «Finis, ut delectet ${ }^{56}$ [sa fin est de plaire] ». Dans l'Église post-tridentine, le cardinal Paoletti, archevêque de Bologne, assignait trois principales fonctions à l'art dans ses Discorsi: "dilettare, insegnare e muovere ${ }^{57}$. Le verbe dilettare vient en premier. Même si elle trouve ses racines à l'époque médiévale, c'est avec l'avènement des Temps nouveaux et de la métaphysique de la subjectivité que semble s'imposer la détermination de l'expérience du beau en termes de délectation.

La promotion de la musique participe éminemment de l'esthétisme. Elle vise à flatter l'ego, celui de chaque individu comme celui de la foule ou de l'État devenu lui aussi «œuvre d'art ${ }^{58}$. Chaque individu est au fond un ego hypertrophié à l'époque de la métaphysique de la subjectivité et de ses avatars, et pas seulement Victor Hugo qui parsema sa maison de Guernesey, «Hauteville House», de l'inscription «EGO HUGO». Il n'est pas jusqu'à la création artistique qui ne soit entièrement absorbée par la faisance en sa calculabilité programmée. L'homme réduit à lui-même est susceptible de se faire lui-même (d'où l'eugénisme, l'hygiène raciale et «l'homme nouveau » des années 1930), ne rencontre partout que lui-même - ce qu'il est convenu d'appeler dans le vocabulaire des philosophes «l'écueil du solipsisme» - dans l'expérience vécue et la griserie où il cherche d'autant

54. C. Baudelaire, «Le peintre de la vie moderne», in Euvres, Paris, Gallimard (Bibliothèque de la Pléiade), 1961, p. 1154.

55. Cité par É. Gilson, Peinture et réalité, Paris, J. Vrin, 1972, p. 230.

56. R. Descartes, Compendium musica, in Euvres, C. Adam, P. Tannery (éd.), Paris, J. Vrin, t. X, 1966, p. 89.

57. Cité par A. Dupront, L'image de religion dans l'Occident chrétien: d'une iconologie historique, Paris, Gallimard, 2015, p. 29.

58. M. Heidegger, GA 95, 150. 
plus désespérément à s'oublier qu'il bute toujours sur lui-même et tourne en rond à l'intérieur de sa propre cage dorée, celle de sa propre subjectivité. La suprême jouissance est le se-sentir [Sich-fühlen] - au sens précisément où l'on dit familièrement de quelqu'un qu'il «ne se sent plus»-, se sentir comme sujet: «Dans la mesure où l'homme se sent porté par des sentiments, quelque chose d'autre que lui-même semble venir à sa rencontre du fait de ces sentiments qu'il ressent, et pourtant, ce qu'il peut bien rencontrer en cet autre, ce n'est jamais que - lui-même» 59 .

\section{L'origine de l'œuvre d'art, amorce d'un «autre commencement»?}

C'est demander si l'art pourrait être une ressource à l'âge d'une urgente transition.

Heidegger a donc risqué, à partir de 1935 et de sa Kunstwerk-Abhandlung, une approche radicalement nouvelle de l'art et de l'œuvre d'art visant à surmonter l'esthétique et à préparer une métamorphose de l'être humain en Dasein. La nouveauté de cette approche n'apparaît pleinement que par contraste avec la situation de ce que l'art est devenu « aujourd'hui », à laquelle Heidegger consacre une analyse approfondie. Il faut se garder de minimiser l'importance du fait que les Apports à la philosophie. De l'avenance de 19361938, seconde œuvre majeure de Heidegger en ses huit parties composées de six fugues qui inaugure la pensée de l'histoire de l'être, reviennent en conclusion sur (et surtout à) l'origine de l'œuvre d'art ( $\left.\mathrm{n}^{0} 278\right)$. On peut notamment y lire ceci:

La conception des Temps nouveaux: faire ressortir l'activité, tout ce qu'il a fallu «faire» pour aboutir à une œuvre, le «génie» - et parallèlement: l'« œuvre» comme exploit. Finalement, l'art au fond n'est plus qu'un moyen de la politique culturelle ${ }^{60}$.

L'univers de la «faisance» et de la cause efficiente s'est substitué à celui de la mise en œuvre de la vérité. La mise en œuvre de la vérité entendue comme déclosion ne nous livre pas toutefois un visage qui en fixerait les traits mais qui nous est montré - et même nous regarde, et pas seulement dans le portrait ou l'autoportrait - tel qu'en lui-même aussi il se dérobe. Ce contre-mouvement de la déclosion, ce principe de fermeture ou cette essentielle réserve, Heidegger l'appelle de manière plutôt inattendue, dans

59. M. Heidegger, GA 95, 149 (nous soulignons).

60. M. Heidegger, GA 65, 507; Apports à la philosophie..., p. 575. 
sa conférence de 1935, la terre, celle qui reprend en son sein. Au monde tel qu'il se découvre à la faveur de l'œuvre d'art vient s'opposer le recouvrement de la terre, en un jeu conflictuel qui est aussi partage d'une réciprocité - harmonie héraclitéenne. Dans le litige auquel elle donne lieu entre monde et terre, et qui lui donne sa tension interne, l'œuvre d'art expose et en même temps abrite, Lichtung et Verbergung, non sans donner à voir ce mouvement même par lequel toutes les choses se retirent âprement en elles-mêmes et se reprennent en venant s'abriter dans ce que Mallarmé a pu appeler «une délicatesse native reployée ${ }^{61}$. Juste avant la nomination de la terre dans la conférence de 1935 intervient celle de la $\varphi^{\prime} \sigma ı \varsigma^{62}$, et il est difficile de lire tout ce développement sans songer au fragment 53 d'Héraclite si souvent

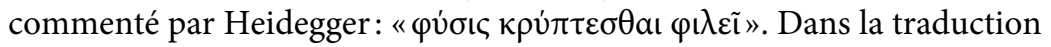
due à Jean Beaufret: «Rien n'est plus propre à l'éclosion que le retrait».

Par-delà ou plutôt en deçà de toutes les interprétations auxquelles l'art a $\mathrm{pu}$ donner lieu pour autant qu'elles relèvent $\mathrm{du}$ "premier commencement » et demeurent tributaires de la métaphysique, Heidegger tente de se mettre à l'écoute de l'œuvre d'art, de se laisser visiter ou interpeller par elle. En cela il demeure rigoureusement fidèle à la méthode phénoménologique comme au retour qu'elle préconise aux choses mêmes ${ }^{63}$. Suite à toutes les interprétations en termes de symboles et d'allégories, voire de "messages ", auxquelles sa toile Guernica a pu donner lieu, Picasso a pu déclarer:

Ce taureau est un taureau et ce cheval est un cheval. Si vous attribuez une certaine interprétation à certains éléments de mes peintures, il se peut que cela soit tout à fait juste, mais je ne souhaite pas livrer cette interprétation [...]. Je peins pour la peinture. Je peins les choses pour ce qu'elles sont ${ }^{64}$.

Ces deux dernières phrases résument peut-être toute l'ambiguïté que nous avons tenté de dégager ici dans la situation de l'art d'aujourd'hui, où Heidegger voit plutôt une absence de site: n'y a-t-il pas en effet une légère différence entre peindre pour la peinture et peindre les choses pour ce qu'elles sont? À moins qu'il ne faille voir dans cette formulation de Picasso l'expression d'un dédoublement constitutif de ce que Cézanne appelait «la vérité en peinture».

Il semble y avoir une divergence d'appréciation entre Heidegger et Jean Beaufret sur le "phénomène» Picasso. Si Cézanne est bien aux yeux de Jean

61. S. Mallarmé, cité par H. Mondor, Vie de Mallarmé, Paris, Gallimard, 1941, p. 674.

62. M. Heidegger, GA 5, 28; Chemins..., p. 45.

63. F.-W. von Herrmann, Heideggers Philosophie der Kunst..., p. 13.

64. Cité par R. Arnheim, Picasso's "Guernica”, Berkeley - Los Angeles - New York, University of California Press, 2006, p. 138. 
Beaufret "plus essentiel et plus profond encore que ce qui glorieusement lui fait suite", et qui en fut le "radieux déclin", cela "ne veut nullement dire» pour autant, ajoute-t-il, «que Braque et Picasso soient moindres que Cézanne», qui leur fut "germinal» ${ }^{65}$. Mais lorsque Heidegger évoque en 1958 à Aix-en-Provence "le chemin de Cézanne, auquel, de son début jusqu'à sa fin, [son] propre chemin de pensée répond à sa façon ", est-ce à dire que "Cézanne, c'était pour lui [= Martin Heidegger] la voie ouverte vers deux sommets, Braque et Picasso " ${ }^{66}$ ? Il est permis d'en douter, même si ce propos de Jean Beaufret demeure d'une foncière ou merveilleuse ambiguité, comme on voudra dire, et sans doute parfaitement voulue, selon que l'on met l'accent sur l'ouvreur, le "primitif» de sa "propre voie» que Cézanne disait être, ou bien sur les deux «sommets» auxquels cette ouverture aura permis de culminer.

On sait en effet, notamment depuis la parution de l'ouvrage de Petzet en 1983, que Heidegger a émis des réserves vis-à-vis de Picasso. Heidegger lui-même a pu déclarer en 1958, donc l'année même de sa conférence aixoise mais aussi dix ans après ce que nous apprennent les "Cahiers» dans l'état actuel de leur publication:

Je tiens Paul Klee en plus haute estime que Picasso. À mes yeux, Paul Klee est un peintre plus important que Picasso ${ }^{67}$.

Libre à Heidegger, dira-t-on, de préférer tel peintre à tel autre. Chacun ses goûts! Mais si la "préférence» ainsi affichée n'était qu'affaire de goût personnel, elle n'aurait d'intérêt que purement biographique, voire anecdotique. Elle nous renseignerait peut-être sur le «vécu» de Heidegger face aux œuvres de ces deux maîtres - à supposer quelque chose de tel - mais ce serait là sans doute la plus mauvaise façon d'entrer dans les considérations de Heidegger sur l'art et demeurer sourd à tout ce qu'il a pu dire de l'expérience vécue comme faisant écran à l'œuvre d'art. La haute «estime » dans laquelle Heidegger tient Paul Klee, qu'il semble avoir découvert au cours des années 1950, l'«importance» qu'il lui accorde, sont décidément à comprendre tout autrement. Pourquoi Heidegger tient-il Paul Klee pour plus estimable et plus considérable ou important que Picasso, plus estimable parce que plus important? Pourquoi apprécie-t-il davantage sa peinture que celle de Picasso? En toute rigueur, la "préférence» ainsi affichée ne

65. J. Beaufret, Dialogue avec Heidegger, Paris, Minuit, t. III, Approche de Heidegger, 1974, p. 155-156.

66. Ibid. (nous soulignons). Parmi ces «sommets» un grand absent, Matisse, dont le nom ne figure pas dans le livre de Petzet, mais que Beaufret mentionne comme tel (ibid., p. 121).

67. M. Heidegger, GA 16, 776, \$290. Voir, dans le présent numéro, p. 18. 
peut signifier, suite à tout ce qui vient d'être exposé, que ceci: Paul Klee donne à voir et accomplit, à rebours de Picasso, la mutation de l'art propice à l' "autre commencement».

Sur tous ces points les déclarations de Heidegger sont marquées par une grande prudence. Il déclare par exemple à Petzet reconnaître bien volontiers la «créativité [Künstlerkraft] » de Picasso - «cela ne fait aucun doute " ${ }^{68}$. Il n'est pas nécessaire d'avoir vu beaucoup de tableaux de Picasso pour savoir qu'une incroyable énergie s'en dégage. C'est cette vigoureuse puissance d'ébranlement qui fera dire à Paul Eluard dans Donner à voir: "À partir de Picasso, les murs s'écroulent ${ }^{69}$. Heidegger ajoute toutefois au propos que nous venons de citer: "Mais je ne vois toujours pas si cette créativité est à même de montrer ne serait-ce que le lieu essentiel, celui de l'avenir ${ }^{70}$. Retenons l'indication de «ce lieu essentiel, celui de l'avenir». À savoir celui où se joue l'éventuelle mutation de l'art qui pourrait provoquer à sa façon et, répétons-le, motu proprio, une métamorphose de l'être humain en Dasein. Mutation que Heidegger semble avoir pressentie chez Paul Klee au point, dit-on, de songer à écrire une seconde partie à sa conférence de 1935 sur L'origine de l'œuvre d'art, mais dont on ne sache pas qu'elle ait vu le jour ${ }^{71}$.

À l'arrière-plan de toutes les considérations sur l'art dans les «Cahiers de travail» se tient donc la conférence sur L'origine de l'œuvre d'art, dont le texte opère un certain nombre de déplacements, élargissements et innovations par rapport à la problématique d'Etre et temps. Nombre d'interprètes, et non des moindres, ont vu dans cette inflexion l'écho de la rencontre de la pensée de Heidegger avec la poésie de Hölderlin, auquel la conférence de 1935 laisse d'ailleurs le dernier mot ${ }^{72}$. C'est dans ce sillage que s'inscrivent toutes les considérations sur l'art dans les «Cahiers", comme autant de prolongements et de nouveaux éclairages, souvent inattendus, qui, dans la perspective de l'histoire de l'être, voient dans l'art la possibilité que s'inaugure une autre histoire, celle d'un «autre commencement» dont les précurseurs sont à vrai dire déjà là, qu'ils s'appellent Hölderlin ou encore

68. H. W. Petzet, Le chemin de l'étoile..., p. 168-169.

69. Cité par J. Beaufret, De l'existentialisme à Heidegger, p. 97 (note 1).

70. H. W. Petzet, Le chemin de l'étoile..., p. 168-169.

71. Mais ce pourrait bien être une légende. Voir à ce sujet G. Seubold, «Heideggers nachgelassene Klee-Notizen", Études heideggeriennes, vol. 9, 1993, p. 5; voir aussi S. Peetz, "Welt und Erde. Heidegger und Paul Klee», Études heideggeriennes, vol. 11, 1995, p. 167-186.

72. Voir H.-G. Gadamer, «Zur Einführung», in M. Heidegger, Der Ursprung des Kunstwerkes, Stuttgart, P. Reclam, 1960, p. 99; W. Biemel, Heidegger, Hambourg, Rowohlt, 1973, p. 81; F.-W. von Herrmann, Heideggers Philosophie der Kunst..., p. 372. Voir aussi S. Zimmermann, «Heidegger über den Streit von Welt und Erde», Études heideggeriennes, vol. 33, 2017, p. 209, qui voit dans l'introduction du concept de terre «l'innovation véritable et décisive de la Kunstwerk-Abhandlung». 
Caspar David Friedrich ${ }^{73}$, qui ne sont pas derrière nous mais devant nous, à venir en leur énigmatique contemporanéité et proximité. Que Heidegger ait pu songer à écrire une seconde partie à sa conférence sur L'origine de l'ouvre d'art, mais cette fois à la lumière de Paul Klee, sans être pour autant à l'affût des «dernières nouveautés» - ou que du moins l'on ait pu lui prêter cette intention -, indique assez à quel point l'art a pu demeurer pour lui une énigme qu'il aura tout fait pour donner à voir.

Envisager l'art comme ressource à l'âge d'une urgente transition ne signifie pas puiser dans les merveilles de l'art du passé des consolations dans l'attente de jours meilleurs, ni non plus lui élever un mausolée à la manière d'André Malraux, mais bien affronter notre présent et guetter en lui les signes d'une mutation de l'art susceptible d'éveiller l'être humain à lui-même - ou plutôt à soi-même - dans sa manière d'être au monde. À la mise en ouvre de la vérité en sa déclosion qu'est l'œuvre d'art répond ainsi la remise en cause tout aussi radicale de qui nous sommes en même temps qu'un aperçu de celles et ceux que nous pourrions être de préférence.

Pascal David

73. M. Heidegger, GA 95, 364 . 
